\title{
Between-dimension flanker effects: A clarification with encouraging implications
}

\author{
J. TOBY MORDKOFF \\ Pennsylvania State University, University Park, Pennsylvania
}

\begin{abstract}
Models of selective and divided attention have evolved to be remarkably similar (e.g., Cohen \& Shoup, 1997; Mordkoff \& Yantis, 1993). One remaining difference between these models is the inclusion of all-or-none gates within a recent model of selective attention; the existence of these gates is supported by the absence of a between-dimension flanker effect. However, the existence of these gates is also inconsistent with a recent model of divided attention. In the present experiment, the evidence in favor of all-or-none gates was explored, with the following result: When the task involves colors and letters as the stimuli, and when display features are presented at equal rates, no evidence of these gates is observed. Although other issues remain to be resolved, these findings bode well for the general goal of a unified model of divided and selective attention.
\end{abstract}

Selective attention is what enables people to respond to task-relevant information while simultaneously ignoring task-irrelevant information. Divided attention is what enables people to respond to more than one piece of information at the same time. The experimental tasks used to study these two abilities are usually quite different. Yet despite this difference, recent models of selective and divided attention have adopted similar architectures; in fact, these models have begun to resemble each other to a remarkable degree (compare, e.g., Cohen \& Shoup, 1997, Figure 12, with Mordkoff \& Yantis, 1993, Figure 6). In particular, models concerned with each ability have incorporated the idea that the visual system includes separate modules for processing color and shape, and that the outputs from these modules are combined by postperceptual processes. This type of architecture enjoys considerable support from a wide variety of experimental paradigms (see, e.g., DeYoe \& Van Essen, 1988; Garner, 1978; Treisman, 1988). This convergence across paradigms is encouraging, since the long-term goal of theorists working in both areas would seem to be a general model of information processing, as opposed to a model that can explain only one type of performance. ${ }^{1}$

However, while models of selective and divided attention have grown to be more similar, at least one important difference has remained. The model of divided attention presented by Mordkoff and Yantis (1993) explicitly includes the claim that the outputs from the color- and shapeprocessing modules are summed before coming to activate an overt response; in this way, partial information

This research was supported by a grant from Pennsylvania State University. I thank Rich Carlson, Marc Grosjean, Cathleen Moore, Tram Neill, Richard Schweikert, and two anonymous reviewers for their useful comments, and Justin Silverman for his help in conducting the experiment. Send correspondence to J. T. Mordkoff, Department of Psychology, 621 Moore Bldg., Penn State University, University Park, PA, 16803 (e-mail: jtm 12 (apsu.edu). about the colors in a display is combined with partial information about the shapes in a display. This is an example of coactivation (see Miller, 1982). In contrast, the model of selective attention presented by Cohen and Shoup (1997) includes all-or-none gates between the color and shape modules and the units that activate overt responses; this precludes the possibility that partial information concerning color can be directly combined with partial information concerning shape. Only a complete target code is allowed to pass through an all-or-none gate.

The present study was designed to explore this difference. In particular, the experiment was designed to examine the evidence that supports the inclusion of allor-none gates within the model of selective attention. If it can be shown that the transmission of information from perceptual modules to response processes does not always occur in an all-or-none manner, then one of the few direct discrepancies between models of selective and divided attention will have been removed.

\section{Evidence of Information Gating \\ in Selective Attention}

The evidence cited in support of the all-or-none gates between the separate perceptual modules and the units that activate responses is both elegant and simple. An experiment reported by Cohen and Shoup (1997) included an ingenious modification to the standard flankers task (B. A. Eriksen \& C. W. Eriksen, 1974). In the standard flankers task, two letters are assigned to each of two different responses, and subjects are required to respond to a target presented at fixation while ignoring distractors, called flankers, that are presented to the left and right of the target. On some trials, called compatible, the flankers are letters associated with the same response as is the target. On other trials, called incompatible, the flankers are letters associated with the opposite response as is the target. The typical result is that the identity of the flankers has a significant effect on performance: Compatible trials 
produce faster and more accurate responses than incompatible trials. This is the flanker effect.

Cohen and Shoup (1997) extended this design to create the two-dimensional flankers task. In their first set of experiments, one color and one shape were mapped onto the left-hand response, and another color and another shape were mapped onto the right-hand response. In contrast to most previous work (which has used letters), the specific shapes used by Cohen and Shoup were lines that could be vertical or slanted to the left or right. Thus, for example, some subjects were asked to make a left-hand response if the center stimulus was either green or slanted to the left, and a right-hand response if the target was either red or slanted to the right. The neutral features for these subjects were vertical and blue, so one of four stimuli would be presented in the center location on a given trial: green vertical, blue left-slant, red vertical, or blue right-slant. The first two of these specifics would require a left-hand response; the second two would require a right-hand response. At the same time, task-irrelevant flankers - which were also oriented, colored lines-were presented to the left and right of the target. As in the standard flankers task, the features of the flankers were often associated with the correct or incorrect response.

Like the standard flankers task, the two-dimensional flankers task requires that the subject ignore the stimuli that surround the target. However, in contrast to the standard flankers task, there are two possible relationships between the flankers and the target. The critical feature of the flankers can be from the same dimension as the target's (e.g., the target is red and the flankers are either red or green); these are within-dimension trials. Alternatively, the critical feature of the flankers can be from the opposite dimension as the target's (e.g., the target is red and the flankers are slanted to either the left or right); these are between-dimension trials. Separate measures of the flanker effect are calculated for the within- and betweendimension conditions.

The crucial finding of Cohen and Shoup (1997, Experiment 1) was that the shape of the flankers had no effect on performance when the target was defined by color (and likewise for color flankers and shape targets). In other words, there was no between-dimension flanker effect. At the same time, the shape of the flankers did have an effect on performance when the target was also a shape (and likewise for colors). Replicating a myriad of previous studies (e.g., B. A. Eriksen \& C. W. Eriksen, 1974), there was a within-dimension flanker effect.

It is the absence of a between-dimension flanker effect that supports the existence of all-or-none gates. These gates prevent partial information from one stimulus dimension from reaching the nodes that activate an overt response; only complete information is allowed to move through. Therefore, partial information from one stimulus dimension (e.g., the color of the flankers) cannot affect performance on trials on which the response is determined by the opposite dimension (e.g., when the center stimulus has a target shape). In this way, the all-or-none gates explain why no between-dimension effects are observed. At the same time, the existence of all-or-none gates does not preclude the possibility of within-dimension effects, because these can occur within a perceptual module.

\section{Alternative Explanations of the \\ Evidence of Information Gating}

The finding of a null between-dimension effect may seem somewhat surprising. First, there is the evidence from divided-attention tasks that colors and shapes can coactivate a response (Mordkoff, Miller, \& Roch, 1996; Mordkoff \& Yantis, 1993); coactivation is not possible when responses are activated in an all-or-none manner, because this type of information transmission does not allow two codes to be combined prior to reaching some criterion. Second, there is the evidence in favor of continuous models for this type of task (e.g., Coles, Gratton, Bashore, C. W. Eriksen, \& Donchin, 1985; C. W. Eriksen \& Schultz, 1979), whereas an all-or-none gate would seem to be the antithesis of continuous flow.

One possible explanation for the evidence of all-ornone gates in selective attention would focus on the specific stimuli employed in the experiments cited above. The divided-attention tasks (e.g., Mordkoff \& Yantis, 1993) have all involved letters as the shape-defined stimuli, as have the experiments that support a continuous-flow model for selective attention (e.g., Coles et al., 1985). In contrast, the experiments in which null between-dimension effects (Cohen \& Shoup, 1997) were found used oriented lines. Thus, it remains possible that the evidence supporting the existence of all-or-none gates is unique to tasks that involve only very simple shape features.

Cohen and Shoup (1997) addressed this possibility by conducting experiments involving two different classes of alphanumeric characters as the two separate "dimensions." These produced very different results from those that had involved colors and oriented lines - for example, letter flankers were shown to affect performance on trials where the target was a digit (and vice versa) - prompting the conclusion that it is not the type of shape features that determines whether a between-dimension effect is observed; rather, it is the specific mixing of the color and shape dimensions that causes between-dimension effects to disappear. However, they did not conduct the more direct test. In particular, Cohen and Shoup did not report any experiments in which colors and letters were the two classes of stimuli. The present study fills in this small gap.

Another difference between the studies of selective and divided attention concerns the inclusion (within the experimental designs) of certain correlations, called "biased contingencies." These contingencies are created by differences in the frequency of various stimulus displays and can have profound effects on performance (see, e.g., Miller, 1987; Mordkoff, 1996; Mordkoff \& Miller, 1993; Mordkoff \& Yantis, 1991). The experiments concerning color and shape divided attention (Mordkoff \& Yantis, 1993) used designs that did not include any biased contingencies; all displays and all features appeared on the same 
number of trials. In contrast, a subtle contingency exists within the design used to explore within- and betweendimension flanker effects (Cohen \& Shoup, 1997).

In the design used by Cohen and Shoup (1997, Experiment 1), there was only one neutral color and only one neutral shape. Because of this, each neutral feature was presented at the center location on half of the trials. (For example, on half of all trials, the center stimulus had a target color - and there were no redundant-target trials--so the shape of the center stimulus was neutral.) In contrast, each target feature only appeared in the center location on one fourth of the trials. Thus, neutral features appeared in the center location on twice as many trials as did target features. If mechanisms sensitive to this difference in display-feature frequency exist (and there is evidence from divided attention that they do), this difference could act to reduce the size of the between-dimension flanker effect.

\section{The Present Experiment}

In most respects, the design of this experiment parallels that used by Cohen and Shoup (1997, Experiment 1). One color and one letter were both assigned to the lefthand response, and one color and one letter were both assigned to the right-hand response. There were five main conditions, defined by the relationship between the target (presented at fixation) and the two identical flankers (presented to the left and right of fixation). For illustration, assume that the left-hand targets are red and $X$, whereas the right-hand targets are green and $O$. Example displays for this mapping include (with the critical features in italics): (1) neutral—red $\mathrm{M}$ surrounded by blue Ms; (2) within-dimension compatible-red $\mathrm{M}$ surrounded by red Ms; (3) within-dimension incompatible-red $\mathrm{M}$ surrounded by green Ms; (4) between-dimension compatible - red M surrounded by blue $X \mathrm{~s}$; and (5) betweendimension incompatible-red $\mathrm{M}$ surrounded by blue $O$ s.

Besides the manipulation of flanker-target compatibility, the present study included a second factor involving the number of neutral features. This manipulation was included to explore the effects of differences in displayfeature frequency. For one group of subjects, there was only one neutral feature per dimension, replicating Cohen and Shoup (1997, Experiment 1). This condition included a display-feature frequency bias, in that neutral features appeared at the center location on twice as many trials as did target features. For the other group of subjects, there were two neutral features per dimension. In this condition, all features appeared at the center location on the same number of trials.

\section{METHOD}

A total of 40 undergraduates from Pennsylvania State University participated. 20 in each group. All were naive as to the study's design and purpose. Each was assigned a random mapping of colors and letters from the set of red, green, blue, and yellow, and $A, I, M$, and $O$. For all subjects, one color and one letter were assigned to the left response (made with the left index finger), and one color and one letter were assigned to the right response (made with the right index finger). For the subjects in the one-neutral-feature group, only one of the remaining two colors and one of the remaining two letters were used. For the subjects in the two-neutral-features group, the remaining two colors and two letters were each used equally often.

Each letter subtended visual angles of $1.15^{\circ}$ (height) and $0.75^{\circ}$ (width). The center of each of the flankers was $1.25^{\circ}$ to one side of fixation. The fixation point was a small white cross $\left(0.50^{\circ} \times 0.50^{\circ}\right)$. Fixation was presented for $500 \mathrm{msec}$, followed by a 350 -msec blank screen, and then by the trial display until a response was made. The intertrial interval was $1,500 \mathrm{msec}$.

The five main conditions - within-dimension compatible, withindimension incompatible, between-dimension compatible, betweendimension incompatible, and neutral-occurred equally often. The target was defined by color on half of the trials; the target was defined by shape (letter) on the other half of the trials. The left- and right-hand responses were each correct on half of the trials.

Experimental sessions began with written instructions and four blocks of practice. During these blocks, the assignment of targets to responses was displayed (again) following every error. After practice, there were 10 blocks of testing trials. During testing, only a beep was presented following an error $(500 \mathrm{~Hz}, 100 \mathrm{msec})$. Complete feedback, including accuracy and mean response time, was given at the end of each block during an enforced, 7 -sec break. There were 40 trials in each block, preceded by three warm-up trials that were never analyzed.

\section{RESULTS}

To avoid having to interpret higher order interactions, the data were analyzed in terms of the size of the flanker effect, which is defined as the difference (in mean response time) between the compatible and the incompatible conditions. The data from the neutral condition, therefore, were not analyzed, but are reported in Table 1. (The more complicated, complete analysis-including the neutral condition-supports the same conclusions.) Separate measures of the flanker effect were calculated for the within- and between-dimension conditions. Thus, the data were analyzed in terms of two factors: flankertarget dimensional relationship - whether the critical feature of the flankers was from the same or the opposite dimension as the critical feature of the target - which had levels within and between; and number of neutral features per dimension, which had levels one and two. The response time results are shown in Figure 1. Complete details on the mean response times and error rates are given in Table 1. Errors were very rare (and positively correlated with mean response time across conditions; $r=.43$ ), so they were not subjected to any systematic analysis; all of what follows concerns mean response time. ${ }^{2}$

There was a significant interaction between flankertarget dimensional relationship and number of neutral features per dimension $[F(1,38)=4.12, p<.05]$ (see Figure 1). Therefore, separate analyses of the within- and between-dimension effects were conducted for each of the two groups of subjects using two-tailed $t$ tests.

For the one-neutral-feature group, there was a significant difference between the within- and betweendimension flanker effects $[t(19)=2.40, p<.05]$. How- 
Table 1

Mean Response Time and Error Rate by Flanker-Target Dimensional Relationship, Number of Neutral Features, and Target Dimension

\begin{tabular}{|c|c|c|c|c|c|c|c|c|}
\hline & \multicolumn{4}{|c|}{ One Neutral Feature per Dimension } & \multicolumn{4}{|c|}{ Two Neutral Features per Dimension } \\
\hline & \multicolumn{2}{|c|}{ Color Target } & \multicolumn{2}{|c|}{ Letter Target } & \multicolumn{2}{|c|}{ Color Target } & \multirow{2}{*}{$\frac{\text { Letter }}{M}$} & \multirow{2}{*}{$\begin{array}{r}\text { Target } \\
\% \mathrm{E} \\
\end{array}$} \\
\hline & $M$ & $\% \mathrm{E}$ & $M$ & $\% \mathrm{E}$ & $M$ & $\% \mathrm{E}$ & & \\
\hline \multicolumn{9}{|c|}{ Within-Dimension } \\
\hline Compatible & 529 & 2.7 & 515 & 1.4 & 533 & 2.8 & 514 & 1.2 \\
\hline Incompatible & 556 & 2.9 & 547 & 2.3 & 547 & 3.0 & 533 & 2.5 \\
\hline \multicolumn{9}{|c|}{ Between-Dimension } \\
\hline Compatible & 537 & 2.5 & 523 & 2.0 & 535 & 1.9 & 524 & 1.3 \\
\hline Incompatible & 551 & 3.4 & 535 & 1.4 & 553 & 3.0 & 535 & 1.9 \\
\hline Neutral & 530 & 2.0 & 513 & 1.3 & 538 & 2.3 & 515 & 0.5 \\
\hline
\end{tabular}

ever, when each flanker effect was compared separately with zero, it was found that both were reliable $[t(19)=8.86$ and 2.31 for within- and between-dimensions, respectively; $p<.001$ and $p<.05$, respectively].

For the two-neutral-features group, there was no significant difference between the within- and betweendimension flanker effects $[t(19)=0.22]$. Yet, to parallel the above, separate analyses of each flanker effect were conducted, showing that both were reliable $[t(19)=2.88$ and 3.75 for within- and between-dimensions, respectively; $p<.01$ and $p<.005$, respectively].

\section{DISCUSSION}

The results from this experiment can be summarized in two points First, when highly discriminable colors and letters are used in a twodimensional flankers task, and all features appear at the center location on the same number of trials, the between-dimension flanker effect is as large as the within-dimension effect. This particular finding is new. Second, when neutral features appear at the center location on twice as many trials as do target features, the within-dimension flanker effect is larger than the between-dimension effect. This partially replicates and extends the results found with colors and oriented lines (Cohen \& Shoup, 1997, Experiment 1).

The conclusions that are supported by this pattern of results would seem very encouraging to any theorist seeking to develop a unified model of selective and divided attention. Of primary interest is the finding of a significant between-dimension flanker effect. This demonstrates that the transmission of information from the perceptual modules for color and shape to the units that activate responses does not always occur in an all-or-none manner. (It is the finding of a null betweendimension effect that supports the inclusion of all-or-none gates.) Even stronger: for the situation involving colors, letters, and an experimental design that is balanced in terms of display frequency, there is no evidence of any gating at all; under these conditions, the between-dimension effect is as large as the within-dimension effect. This is the same set of conditions for which previous experiments concerning divided attention have produced evidence of coactivation (e.g., Mordkoff et al., 1996; Mordkoff \& Yantis, 1993). Because all-or-none gating is inconsistent with coactivation, the present evidence against gating is important to the long-term search for a unified model.

Of secondary interest-but no less encouraging - is the observed influence of neutral-feature frequency on the relative sizes of the withinand between-dimension flanker effects. Similar influences of bias or lack of balance in an experimental design have previously been observed in several studies of divided attention (e.g., Mordkoff \& Miller, 1993 Mordkoff \& Yantis, 1991), as well as some other studies of selective attention (e.g., Miller, 1987; Mordkoff, 1996). More generally, these re- sults highlight several of the known similarities between divided and selective attention. Both types of task have provided evidence that color and shape are processed by separate perceptual modules; both have provided evidence that multiple sources of information can activate a single response; and both have provided evidence that display-feature frequency has an effect on performance.

\section{Other Evidence of Information \\ Gating in Selective Attention}

The present study has shown that there is at least one set of conditions under which a selective-attention task does not seem to involve any allor-none gating. However, using another (rather ingenious) multidimensional variant of the basic flankers task, Lavie (1997) has recently provided data that might be taken as evidence of a related type of gating in a similar situation. In particular, this work has shown that responserelated colors and shapes can sometimes have no observable effect on the processing of a fixated color-shape conjunction when these distractor features appear in known, task-irrelevant locations. As a concrete example, assume that a purple-cross target requires a left response, a green-circle target requires a right response, and the experiment is designed so that both features must be detected before a response can be made. Under these conditions, the presence of a purple flanker has no effect on response time unless a cross-shaped flanker is simultaneously present, and a cross-shaped flanker has no effect unless a purple flanker is also present (and likewise for green and circle). In summary, when the task requires the detection of color-shape conjunctions, only the presence of both attributes of a target (in one or more flanking locations) will cause a flanker effect.

These findings are relevant to the present conclusions because they show that the flankers in a multidimensional task can sometimes fail to have any effect on performance. In fact, on some interpretations, these results could be taken as evidence of all-or-none gating in selective attention. However, as pointed out by Lavie (1997), "the imposed tasks forced adherence to an 'and-gate' rule" (p. 554), in that the correct response was determined by the combination of color and shape (contrasting with the task used in the present study), so this conclusion will be probably be limited to tasks involving color-shape conjunctions. Furthermore, the "and-gate" that is required to perform a conjunction task need not operate in an all-or-none manner, because an "and-gate" that works by multiplying the two inputs (such that the output is always zero as long as one input is zero) would also be sufficient.

Other evidence of gating in selective attention might be derived from the present finding that the number of neutral features can affect the results in a two-dimensional flankers task. One potential explanation for this effect (suggested by Tram Neill) is that the neutral feature of a target stimulus can act as a cue concerning which dimension contains the target information. Assume, for example, that the target stimulus is a red $M$ (where red is assigned to the left-hand response and $M$ is neutral) and the flankers are blue $O$ s (where blue is neutral and $O$ is assigned to the right-hand response); this is a between-dimension, incompatible trial. If the letter $\mathrm{M}$ is identified quickly, the optimal use of this infor- 


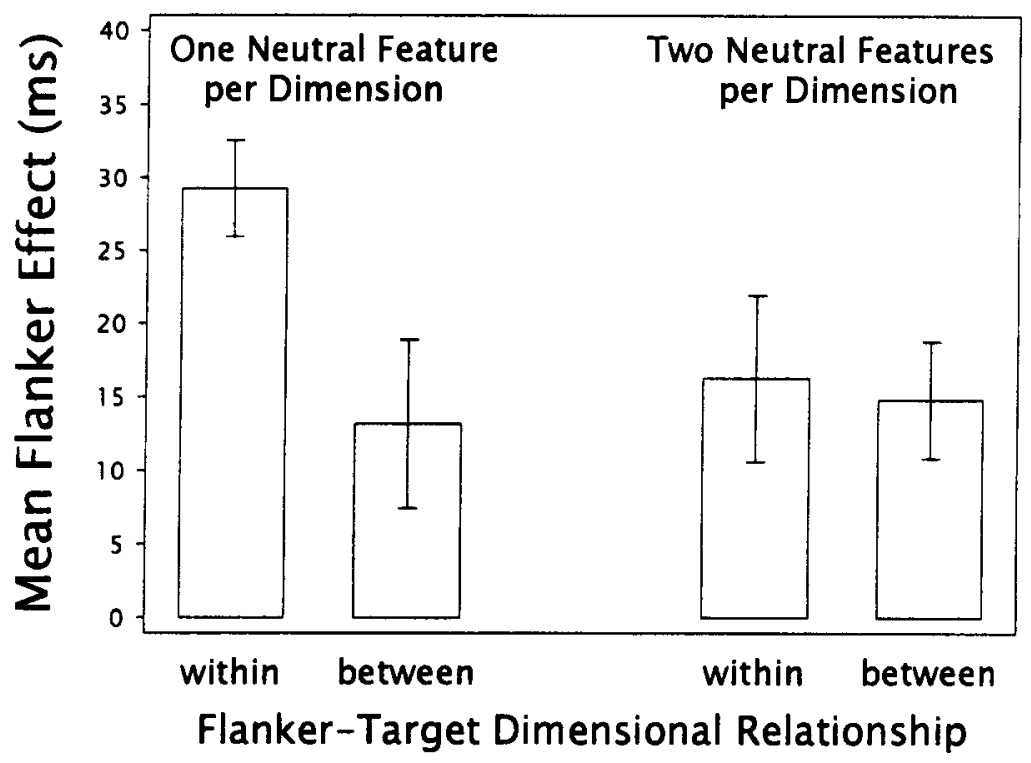

Figure 1. Mean flanker effect (with standard errors of the mean effect) as a function of flanker-target dimensional relationship (within- vs. between-dimensions) and number of neutral features per dimension (one vs. two).

mation would be to "shut off" the shape-processing system, because the presence of a neutral shape in the center location means that the relevant dimension on this trial must be color. More generally, the rapid identification of a neutral feature in the center location could be used to close a gate, causing a reduction or elimination of the betweendimension flanker effect. In contrast, the operation of these putative gates would not have any influence on the within-dimension effect, because preliminary information concerning a neutral feature in the center location on a within-dimension trial would only serve to close the gate from a completely irrelevant perceptual module (i.e., one that contains no features that are associated with either response).

When applied to the present experiment, the ideas sketched above provide the base for a potential explanation of the effect of the number of neutral features on the size of the between-dimension flanker effect. The key issue is whether the neutral feature of the center stimulus is identified before the target feature. When there is only one neutral feature per dimension, such that neutral features appear twice as often as do target features, the probability that the neutral feature will be identified before the target feature could well be quite high. This, in turn, could increase the probability of dimensional gating. In contrast, when there are two neutral features per dimension, such that neutral features appear at the same frequency as do target features, the neutral feature of the center stimulus might not be identified quickly enough for gating to have an effect. Therefore, only under an experimental design that includes a bias in favor of neutral features will one observe a diminished (or absent) between-dimension flanker effect. Conversely, under designs that are not biased in favor of neutral features, no effective dimensional gating will occur, and the within- and between-dimension effects will be equal. 3

\section{Conclusion}

For tasks that involve highly discriminable colors and letters and use experimental designs that are balanced in terms of display-feature frequency, the existing evidence supports two very similar models of selective and divided attention. This seems to be cause for encouragement. Although several issues remain to resolved-including the possibility of gating in similar tasks and the specific mechanism by which display frequency affects selective attention-it does not seem unreasonable to predict that a unified model for both tasks is now within the reach of theorists studying these two different types of information processing.

\section{REFERENCES}

Cohen, A., \& Shoup, R. (1997). Perceptual dimensional constraints in response selection processes. Cognitive Psychology, 32, 128-181.

Coles, M. G. H., Gratton, G., Bashore, T. R., Eriksen, C. W., \& DonCHIN, E. (1985). A psychophysiological investigation of the continuous flow model of human information processing. Journal of $E x$ perimental Psychology: Human Perception \& Performance, 11, 529553.

DEYoE, E. A., \& VAN Essen, D. C. (1988). Concurrent processing streams in monkey visual cortex. Trends in Neurosciences, 11, 219-226.

ERIKSEN, B. A., \& ERIKSEN, C. W. (1974). Effects of noise letters upon the identification of a target letter in a nonsearch task. Perception \& Psychophysics, 16, 143-149.

ERIKSEN, C. W., \& Schultz, D. W. (1979). Information processing in visual search: A continuous flow conception and experimental results. Perception \& Psychophysics, 25, 249-263.

Garner, W. R. (1978). Aspects of a stimulus: Features, dimensions, and configurations. In E. Rosch \& B. B. Lloyd (Eds.), Cognition and categorization (pp. 99-133). Hillsdale, NJ: Erlbaum.

LAviE, N. (1997). Visual feature integration and focused attention: Response competition from multiple distractor features. Perception \& Psychophysics, 59, 543-556.

Miller, J. (1982). Divided attention: Evidence for coactivation with redundant signals. Cognitive Psychology, 14, 247-279.

Miller, J. (1987). Priming is not necessary for selective-attention failures: Semantic effects of unattended, unprimed letters. Perception \& Psychophysics, 41, 419-434.

MordkofF, J. T. (1996). Selective attention and internal constraints: There is more to the flanker effect than biased contingencies. In A. Kramer, M. G. H. Coles, \& G. Logan (Eds.), Converging operations in the study of visual selective attention (pp. 483-502). Washington, DC: American Psychological Association.

Mordkoff, J. T., \& Miller, J. (1993). Redundancy gains and coactivation with two different targets: The problem of target preferences 
and the effects of display frequency. Perception \& Psychophysics, $\mathbf{5 3}$. 527-535.

MordkofF, J. T., Miller, J., \& Roch, A.-C. (1996). Absence of coactivation within the motor component: Evidence from psychophysiological measures of target detection. Journal of Experimental Psychology: Human Perception \& Performance, 22, 25-41.

MoRdkofF, J. T., \& YanTIS, S. (1991). An interactive race model of divided attention. Journal of Experimental Psychology: Human Perception \& Performance, 17, 520-538.

MordKoff, J. T., \& YantIS, S. (1993). Dividing attention between color and shape: Evidence of coactivation. Perception \& Psychophysics, 53, 357-366.

Treisman, A. (1988). Features and objects: The fourteenth Bartlett Memorial Lecture. Quarterly Journal of Experimental Psychology, 40A, 201-237.

\section{NOTES}

1. The term divided attention is here used in a somewhat restricted sense, referring only to situations in which people are presented with multiple pieces of task-relevant information but are required to make only one response (e.g., the redundant-target detection task). Similarly, the term selective attention is here used to refer only to situations in which people are required to respond to exactly one stimulus (e.g., the flankers task). Therefore, the present discussion and conclusions should be seen as limited, in that all dual-task situations, for example, are specifically excluded.

2. The reported analysis also ignores the fact that half of the trials involved a color target, and half involved a letter target. This was planned for simplicity and follows previous work (e.g., Cohen \& Shoup, 1997). A separate, unreported analysis yielded a main effect, but no reliable interactions involving the color- versus letter-target factor.

3. It should be noted that this explanation of the effect of the number of neutral features is both speculative and incomplete. Most important: While this idea can explain why the relative sizes of the within- and between-dimension effects are influenced by neutral-feature frequency and why some experiments involving only one neutral feature have failed to produce a between-dimension effect (e.g., Cohen \& Shoup, 1997, Experiment 1), it cannot explain why the present results showed a change in the within-dimension effect, and not the between-dimension effect. The "conditional-gating" model sketched here would predict changes in the between-dimension effect (only).

(Manuscript received October 9, 1997; revision accepted for publication February 26, 1998.) 